\section{MS06 O4}

Protein crystallization on nucleating surfaces inspired by biomineralization S. Fermani, G. Tosi, N. Roveri and G. Falini Department of Chemistry, Alma Mater Studiorum University of Bologna, Italy.

E-mail: giuseppe.falini@unibo.it

\section{Keywords:protein, nucleation, surface chemistry}

The production of diffraction quality protein crystals remains one of the greatest impediments facing structural biologist today: it has always been a bottleneck to structure determination. The Bologna group had designed protein crystal nucleating substrates inspired by the studies on the biomineralization processes [1]. In biomineralization is well known the crystal surfaces are able to recognize and organize specific macromolecules, thus having potential nucleating properties. In the proposed protein crystal growth strategy the control processes over the crystal nucleation and growth occur by means of surfaces functionalized with specific ionisable groups and controlled hydrophilicity. Experimentally the traditional hanging-drop vapour diffusion technique was modified introducing between the glass cover slip and the drop, a surface opportunely functionalised with ionizable chemical groups. The used nucleant surfaces were silanized mica and sulphonated polystyrene films. Studies of contact angles, roughness, morphology (AFM, SEM) and ATR-FTIR demonstrated that both surfaces are substrates suitable for macromolecular crystallization: they are enough smooth to avoid topographic effects during nucleation, and their hydrophilicity still allows the deposition of a spherical drop.

To evaluate the nucleant capacity of surfaces and understand the mechanism by which the surfaces control the protein crystallization, several experiments were carried out using three different "model proteins": Thaumatin, Concanavalin A and Lysozyme [2]. The results showed that functionalised surfaces, when compared with siliconized glass cover slip (control), decreased the induction time and the starting protein concentration for the crystal nucleation and growth. An increase of the nucleation density with the increase of the ionisable group density on the surface was also observed. It is proposed that non-specific attractive and local interactions between the charged residues of the protein and the ionisable groups on surface might promote molecular collisions raising the probability of crystalline nucleus formation.

[1] Falini G., Fermani S. Tissue Engineering, 2004, 10(1), 1-6.

[2] Falini G., Fermani S., Conforti G., Ripamonti A. Acta Cryst. D., 2002, D58 (10), 1649-1652.

\section{MS06 O5 \\ Enhanced Protein Crystal Diagnostic Imaging with the DETECT-X Microscope Peter Nollert ${ }^{\mathrm{a}}$, Yiping $\mathrm{Xia}^{\mathrm{a}}$,Werner Kaminski ${ }^{\mathrm{b}}$, Laurelin Ward ${ }^{\mathrm{a}}$, Natalie Duncan ${ }^{\mathrm{a}}$, Mark Mixon ${ }^{\mathrm{a}}{ }^{\mathrm{a}}$ Emerald BioSystems, Bainbridge Island, WA, USA; ${ }^{\mathrm{b}}$ Department of Chemistry, University of Washington, Seattle, USA. \\ E-mail: pnollert@emeraldbiosystems.com}

\section{Keywords: imaging, protein crystal, birefringence}

The DETECT-X microscope enhances the appearance of protein crystallization setups, displaying colorless protein crystals as if they were colored. We will show images that demonstrate the unique protein crystal imaging capabilities of the DETECT-X microscope. The DETCT$\mathrm{X}$ microscope is a fully automated polarization microscope that has trans-illumination and UV-epi illumination options. Protein crystallization trials in traditional crystallization trays can be imaged in an automatic fashion. In its birefringence microscopy mode the DETECT-X microscope produces quantitative images of crystals depicting the orientation of the slow optical axis of protein crystals and their orientation independent, quantitative birefringence.

The combination of oblique angle illumination, quantitative birefringence imaging and UV fluorescence microscopy allows to image protein crystals that have strong contrast with respect to their facets as well as the interior body of the crystal. Colorless, transparent protein crystals appear colored. This enhanced contrast allows researchers to (i) see protein crystals through a layer of precipitate, (ii) discern between amorphous precipitate and non-facetted sphaerulites or pre-crystalline matter, (iii) identify crystal twins, (iv) distinguish between protein and salt crystals. 